\title{
ADAPTAÇÃO PARA A LÍNGUA PORTUGUESA E APLICAÇÃO DE PROTOCOLO DE AVALIAÇÃO DAS DISARTRIAS DE ORIGEM CENTRAL EM PACIENTES COM DOENÇA DE PARKINSON
}

\section{Adjustment to the Portuguese and application to patients with Parkinson's disease of protocol within central origin dysarthrias' assessment}

\author{
Angélica Suelen Fracassi ${ }^{(1)}$, Ana Rita Gatto (2), Silke Weber (3), \\ André Augusto Spadotto ${ }^{(4)}$, Priscila Watson Ribeiro ${ }^{(5)}$, Arthur Oscar Schelp ${ }^{(6)}$
}

\begin{abstract}
RESUMO
Objetivo: traduzir e adaptar protocolo desenvolvido por pesquisadores alemães, adequando-o às características fonéticas e linguísticas do português falado no Brasil. Caracterizar os componentes de fala mais alterados na população com doença de Parkinson, comparando-os com grupo de sujeitos normais na mesma faixa etária. Método: realizou-se a tradução e adaptação do protocolo. Posteriormente foram avaliados 21 pacientes com diagnóstico neurológico de Doença de Parkinson nos estágios Hoehn \&Yarh, entre 2 e 3, e 10 sujeitos normais. O protocolo incluía avaliação da respiração, fonação, ressonância, articulação, prosódia e a análise acústica dos parâmetros vocais. Resultados: o protocolo mostrou-se de fácil aplicação clínica. Nos sujeitos com doença de Parkinson foram observadas alterações predominantes na fonação (85,9\%) e articulação (42,9\%). Conclusão: o estudo demonstrou ser o protocolo uma ferramenta eficiente para a avaliação da disartria em pacientes com doença de Parkinson.
\end{abstract}

DESCRITORES: Disartria; Doença de Parkinson; Avaliação

\section{INTRODUÇÃO}

A disartria é definida por um conjunto de alterações resultantes do distúrbio no controle muscular

(1) Médica Generalista da Assistência Médica Ambulatorial Águia de Haia, São Paulo, SP.

(2) Fonoaudióloga do Departamento de Neurologia e Psiquiatria da Faculdade de Medicina de Botucatu, UNESP, Botucatu, SP.

(3) Médica; Professora Doutora do Departamento de Oftalmologia e Otorrinolaringologia da Faculdade de Medicina de Botucatu, UNESP, Botucatu, SP.

(4) Bacharel em Sistemas de Informação, departamento de Neurologia e Psiquiatria da Faculdade de Medicina de Botucatu, UNESP, Botucatu, SP; Doutor em Engenharia Elétrica pela Universidade de São Paulo.

(5) Fonoaudióloga do Departamento de Neurologia e Psiquiatria da Faculdade de Medicina de Botucatu, UNESP, Botucatu, SP.

(6) Médico; Professor Doutor do Departamento de Neurologia e Psiquiatria da Faculdade de Medicina de Botucatu, UNESP, Botucatu, SP.

Conflito de interesses: inexistente do mecanismo da fala devido lesão no sistema nervoso central ou periférico ${ }^{1}$. Pode afetar um ou mais componentes da produção oral, sendo estes a respiração, fonação, ressonância, articulação e prosódia ${ }^{2}$. Dependendo da etiologia neurológica têm-se quadros disártricos distintos.

Na Doença de Parkinson (DP) descrita, inicialmente, pelo inglês James Parkinson em 1817 como uma doença degenerativa progressiva do sistema extrapiramidal, observa-se alterações relacionadas principalmente à fonação, articulação e prosódia, tais como, monotonia de picht e loudness, redução no stress das palavras, imprecisão na produção de consoantes e voz rouco-soprosa ${ }^{3-5}$.

Analisando 200 pacientes com diagnóstico de Doença de Parkinson, pesquisadores observaram que cerca $89 \%$ deles apresentaram desordens vocais, tais como soprosidade, rouquidão, aspereza e tremor. Distúrbios articulatórios foram encontrados, por sua vez, em $45 \%$, dos pacientes ${ }^{6}$.

No diagnóstico dessas alterações, via de regra, o examinador não tem acesso a protocolos bem 
estruturados para investigação das disartrias. Estudos sobre a percepção da inteligibilidade de fala nas disartrias, baseados em observação clínica, concluem que ao compararmos a análise de um profissional bem treinado e um indivíduo que não trabalha na área, ambos apresentam capacidade de percepção das alterações de fala sem diferença estatisticamente significante ${ }^{7}$.

A avaliação clínica da disartria, presente em inúmeras doenças neurológicas pressupõe preferencialmente a análise de parâmetros vocais por meio da percepção auditiva. Provas de repetição de palavras e frases, discurso e produções orais pré-estabelecidas são alguns dos instrumentos utilizados na prática clínica, sem que isso pressuponha a aplicação de protocolo específico ${ }^{8,9}$.

Além da avaliação clínica é igualmente importante para a conclusão diagnóstica da disartria a observação de aspectos objetivos fornecidos por programas específicos de análise acústica da voz. A relação entre as avaliações subjetiva e objetiva fornece maior segurança no diagnóstico, acompanhamento e controle da eficácia terapêutica nos pacientes com tais comprometimentos. O estudo acústico requer a análise de diferentes parâmetros, incluindo frequência fundamental (correlato físico correspondente à altura), duração (tempo de articulação) e intensidade (energia vocal utilizada pelo falante).

$\mathrm{Na}$ língua portuguesa, não dispomos de muitos protocolos clínicos validados para avaliação da disartria em pacientes com doenças neurológicas. Grande parte dos estudos refere-se à análise perceptiva auditiva e objetiva dos parâmetros vocais em sujeitos com diagnóstico de disartria previamente definido ${ }^{10}$. Walshe et al. ${ }^{11}$, e Ackermann e Ziegler $^{12}$, por exemplo, relacionaram aspectos de inteligibilidade e articulação à disartria em grupo de sujeitos disártricos. Outro aspecto que chama atenção é o fato de que esses autores limitam-se a discutir distúrbios isolados da fala e não o seu conjunto. Já Ortiz ${ }^{13}$ aplicou protocolo de disartria baseado na tradução e adaptação de outros protocolos propostos por autores de língua inglesa, possibilitando a análise conjunta dos componentes da produção oral.

O objetivo do presente estudo foi traduzir e adaptar protocolo desenvolvido por pesquisadores alemães, adequando-o às características fonéticas e linguísticas do português falado no Brasil. A partir de sua aplicação, detalhar os componentes de fala mais alterados em grupo de pacientes com Doença de Parkinson nos estágios 2 e 3 de Hoehn \&Yahr ${ }^{4}$.

\section{MÉTODO}

Foi realizada pesquisa científica de caráter original desenvolvida no Hospital das Clínicas da Faculdade de Medicina de Botucatu.

Participaram do estudo 31 sujeitos, com faixa etária variando de 54 a 75 anos. $\mathrm{O}$ grupo com diagnóstico neurológico de Doença de Parkinson foi constituído por 21 sujeitos classificados entre os estágios 2 e 3 de Hoehn \&Yahr ${ }^{4}$. O grupo controle se constituiu de 10 sujeitos sem história ou queixa de comprometimento neurológico que pudesse comprometer a fala.

O protocolo que serviu de base para a presente proposta de análise foi cedido pelo Professor Wolfrang Ziegler, nome do grupo de estudo em Neurofonética da Unidade de Neuropsiquiatria no Instituto Max-Planck Psiquiatric, Bogenhaus, em Munique, Alemanha ${ }^{14}$, desenvolvido no período de 1984 a 1988.

A primeira versão do protocolo para a língua portuguesa foi feita por médico neurologista brasileiro. A tradução reversa foi realizada por médica otorrinolaringologista, procedente de país de língua alemã e a adaptação realizada por fonoaudiólogas do serviço.

Os dados do exame clínico neurológico foram obtidos no exame de rotina do Ambulatório de Doenças do Movimento do Hospital das Clínicas da Faculdade de Medicina de Botucatu - UNESP. O estagiamento da Doença de Parkinson foi estabelecido conforme proposto por Hoehn e Yahr. Foram selecionados somente os que se enquadravam nos níveis 2 e 3 , ou seja, os que apresentavam sinais e sintomas bilaterais, ausência de alterações de ajuste postural evidente, sem necessidade de auxílio em atividades de vida diária. As aplicações do protocolo foram realizadas por um único avaliador.

O protocolo de avaliação incluiu a análise dos seguintes componentes da fala (Anexo 1):

a) Respiração: foi analisada a frequência respiratória, realizando-se a contagem do número de ciclos respiratórios durante um minuto; e o número de palavras por expiração, observadas a partir da contagem numérica inversa espontânea (40 a 1) e durante a fala espontânea.

b) Fonação: foi realizada avaliação perceptivoauditiva da qualidade vocal e análise acústica. Referindo-se a perceptivo-auditiva avaliou-se o tempo máximo de fonação durante a emissão das vogais sustentadas $/ a /$ e $/ \mathrm{i} /$, das fricativas surda e sonora $/ s /$ e $/ z /$ (proporção entre elas) e a fala espontânea. Avaliou-se a qualidade vocal, sensação de frequência e intensidade (pitch e loudness) e características da emissão (ataque vocal e estabilidade). 
Após a avaliação subjetiva foi feita a análise acústica utilizando Programa Análise de Voz 2.3 DSP Instrumentos, desenvolvido por pós-graduando em processamento de sinais da USP - São Carlos ${ }^{15}$. Foram analisados os parâmetros acústicos da voz: frequência fundamental (f0), medidas de perturbação (jitter, shimer), suavidade espectral de resíduo (SFR), suavidade espectral de filtro inverso (SFF), amplitude do pitch (PA) e coeficiente de excesso (EX) (Figura 1). Para obtenção dos dados foi solicitada a emissão sustentada da vogal /a/. A gravação foi realizada em sala com acústica ambiente, porém, sem ruídos demasiados.

c) Ressonância: foi observada a movimentação velar e faríngea pela emissão dos fonemas /a/ e /ã/, alternadamente, e durante a repetição da sílaba / $\mathrm{Ka}$. Tais movimentos foram classificados como ausente, mínimo e adequado. A emissão nasal foi avaliada com o auxílio do espelho milimetrado de glatzel. Foi solicitada a emissão de palavras e frases, classificando-as como normal, hipernasal ou hiponasal, nos níveis de leve a grave.

d) Articulação: caracterizada pela amplitude dos movimentos de lábio, língua e mandíbula com avaliação da constância e velocidade. Foi solicitado que os sujeitos emitissem espontaneamente de forma sobrearticulada o /i/, / $/$ /, e /pa/, e as sílabas $/ \mathrm{Ka} / \mathrm{e} / \mathrm{ta} /$ alternadamente e com velocidade crescente.

Além disso, para avaliar a articulação, foram observadas a fala espontânea, a descrição de figuras e a leitura de palavras isoladas, com pontos e modos articulatórios distintos (plosivas, fricativas, nasais, líquidas, retroflexas e vibrantes, encontros consonantais e vogais), além das diadococinesias.

Cada item foi classificado como normal ou alterado, e a precisão articulatória graduada de inteligível a ininteligível numa escala de 0 a 6 .

e) Prosódia: foi observada, por meio da leitura, durante a fala espontânea e a partir da entonação dada à fala ao emitir frases afirmativas, interrogativas e exclamativas. Para os sujeitos que não apresentavam habilidade de leitura, as frases eram lidas pelo avaliador sem qualquer entonação, visando não induzir a resposta do paciente.

Para quantificar a disartria, cada componente de fala foi graduado a partir de escala de 0 a 6 , sendo o 0 a ausência do distúrbio e 6 um distúrbio grave. Após esta análise a pontuação obtida em cada componente foi somada, classificando a disartria em leve (1-10 pontos), moderada (11- 20 pontos) ou grave (21- 30 pontos).

O projeto foi aprovado pelo Comitê de Ética em Pesquisa Médica - CREMESP, Hospital das Clínicas, UNESP com o número do protocolo

\begin{tabular}{|l|c|l|}
\hline Parâmetro & Unidade & \multicolumn{1}{c|}{ Descrição } \\
\hline F0 & $\mathrm{Hz}$ & $\begin{array}{l}\text { Avalia o número de ciclos do pulso glotal que ocorrem por segundo. O } \\
\text { limiar masculino/feminino, segundo avaliação estatística, foi estipulado } \\
\text { ser 160Hz, não indicando disfonias enquanto o limiar de 250Hz indica } \\
\text { uma alteração nos padrões de normalidade. }\end{array}$ \\
\hline JITTER & $\%$ & $\begin{array}{l}\text { Avalia a perturbação da freqüência do sinal. É calculado pela razão entre } \\
\text { as variações do período e o período médio constantes da amostra } \\
\text { selecionada. Determinou-se como valor de normalidade o limiar de } \%\end{array}$ \\
\hline SHIMMER & $\%$ & $\begin{array}{l}\text { Avalia a perturbação da amplitude do sinal. Calcula-se o parâmetro pela } \\
\text { substituição do período pela amplitude do sinal. Determinou-se o valor do } \\
\text { limiar (15\%) }\end{array}$ \\
\hline PA & Adimensional & $\begin{array}{l}\text { Amplitude do Pitch: a quantificação desta medida corresponde à mediana } \\
\text { da amplitude do segundo pico da autocorreção do sinal residual. }\end{array}$ \\
\hline SFR & Valor & $\begin{array}{l}\text { Coeficiente de Excesso: é uma medida da forma da distribuição de } \\
\text { amplitudes do sinal residual. É uma avaliação da razão entre o } \\
\text { sinal/ruído. o valor estabelecido como limiar é 2,0. }\end{array}$ \\
\hline SFF & dB & $\begin{array}{l}\text { Suavidade Espectral do Resíduo: é uma medida da variação espectral do } \\
\text { sinal, isto é, está relacionado à variação das amplitudes das } \\
\text { componentes espectrais do sinal. o sinal SFR traz informações do } \\
\text { formato do pulso glotal. }\end{array}$ \\
\hline
\end{tabular}

Figura 1 - Parâmetros acústicos da voz: Programa "Análise da voz" 2.3 DSP Instrumentos 
31/2005. Todos os sujeitos incluídos no protocolo de estudo, tiveram ciência e deram consentimento livre e esclarecido.

Os dados foram submetidos à análise estatística com teste "t" de Student para as variáveis que apresentaram distribuição normal e o teste não-paramétrico de Mann-Whitney para as demais variáveis. Para estudo da associação entre as variáveis foi utilizado o teste do "qui-quadrado".

\section{RESULTADOS}

O protocolo de avaliação das disartrias mostrouse de fácil aplicação em pacientes com Doença de Parkinson (Anexo 1), incluindo os componentes da fala: respiração, fonação, ressonância, articulação e prosódia, além da análise acústica da voz.

Ao ser aplicado nos sujeitos do grupo controle e nos sujeitos com diagnóstico neurológico de Doença de Parkinson, observamos que houve diferença estatisticamente significante $(p<0,05)$ apenas em relação à variável tempo máximo de fonação (TMF). Sendo que o grupo com DP apresentou TMF significantemente mais curto que os sujeitos normais, com variação entre 8,2 e 4,2 segundos, e o grupo controle com variação entre 14,9 e 3,4 (Tabela 1).

Como verificado na tabela 2 pode-se observar frequentes alterações na fonação nos indivíduos do Grupo com Doença de Parkinson, constatando-se alteração na intensidade em 13 indivíduos $(61,9 \%)$, presença de ataque vocal em $8(38,1 \%)$, alteração na frequencia em $10(47,6 \%)$ e no tempo máximo de fonação em 14 (66,7\%).

Em relação à análise acústica realizada a partir da emissão sustentada da vogal /a/ observou-se diferença estatística entre os grupos avaliados em relação às variáveis jitter, shimer, Suavidade Espectral do Filtro Inverso (SFF) e Amplitude do pitch (PA) (Tabela 3).

$\mathrm{Na}$ análise do grupo de sujeitos com diagnóstico de Doença de Parkinson relacionado aos componentes da fala, observaram-se alterações significantes na fonação em 18 sujeitos $(85,7 \%)$, seguidas da articulação em 8 sujeitos $(38,1 \%)$ e 7 na prosódia (33,3\%) (Tabela 4), sendo que no grupo controle não se observou alteração nos parâmetros clínicos avaliados.

Tabela 1 - Dados do protocolo clínico referentes aos parâmetros fonação e respiração, nos grupos com Doença de Parkinson (DP) e Grupo Controle

\begin{tabular}{|c|c|c|c|c|}
\hline \multirow{2}{*}{ Variáveis } & \multicolumn{2}{|c|}{ Grupo } & \multirow{2}{*}{ Estatística t } & \multirow{2}{*}{ Valor $p$} \\
\hline & Parkinson & Normal & & \\
\hline Ciclos respiratórios & $20,5 \pm 5,3$ & $16,9 \pm 2,6$ & 3,58 & 0,05 \\
\hline TMF & $8,2 \pm 4,2$ & $14,9 \pm 3,4$ & $-4,39$ & $<0,001$ \\
\hline Tempo de respiração & $16,4 \pm 9,9$ & $23,0 \pm 8,6$ & $-1,81$ & 0,08 \\
\hline Relação S/Z & $1,2 \pm 0,6$ & $1,1 \pm 0,2$ & 0,57 & 0,58 \\
\hline
\end{tabular}

Média e desvio-padrão, estatística t e valor $p$ referentes às variáveis segundo grupo.

Tabela 2 - Porcentagem de alterações vocais nos grupos com Doença de Parkinson (DP) e Grupo Controle

\begin{tabular}{|c|c|c|c|c|c|}
\hline & \multicolumn{4}{|c|}{ Grupos } & \multirow{3}{*}{ Valor $p$} \\
\hline & \multicolumn{2}{|c|}{ Parkinson $(\mathrm{n}=21)$} & \multicolumn{2}{|c|}{ Normal $(n=10)$} & \\
\hline & $\mathrm{N}^{\mathrm{O}}$ & $\%$ & $\mathrm{~N}^{\circ}$ & $\%$ & \\
\hline Intensidade & 13 & 61,9 & - & & 0,001 \\
\hline Ataque Vocal & 8 & 38,1 & - & & 0,023 \\
\hline Frequência & 10 & 47,6 & 1 & 10,0 & 0,04 \\
\hline $\begin{array}{l}\text { Tempo máximo de fonação } \\
\text { (TMF) }\end{array}$ & 14 & 66,7 & - & & 0,0005 \\
\hline
\end{tabular}

Valores e porcentagem dos parâmetros alterados referente aos grupos e valor de $\mathrm{p}$. 
Tabela 3 - Dados de análise acústica

\begin{tabular}{lcccc}
\hline \multirow{2}{*}{ Variáveis } & \multicolumn{2}{c}{ Grupo } & \multirow{2}{*}{ Estatística t } & \multirow{2}{*}{ Valor $\mathbf{p}$} \\
\cline { 2 - 3 } & Parkinson & Normal & & \\
\hline Jitter & $3,4 \pm 3,6$ & $1,0 \pm 0,7$ & 2,15 & 0,04 \\
Shimer & $11,3 \pm 6,9$ & $5,5 \pm 2,4$ & 2,56 & 0,02 \\
SFR & $-6,8 \pm 3,9$ & $-4,6 \pm 7,2$ & $-1,10$ & 0,28 \\
SFF & $-16,2 \pm 1,5$ & $-18,4 \pm 1,7$ & 3,64 & 0,001 \\
PA & $0,28 \pm 0,08$ & $0,35 \pm 0,07$ & $-2,18$ & 0,037 \\
Ex & $61,6 \pm 22,3$ & $69,4 \pm 27,8$ & $-0,84$ & 0,41 \\
FO Feminino & $184,2 \pm 33,9$ & $209,1 \pm 34,1$ & $-1,39$ & 0,19 \\
FO Masculino & $130,4 \pm 22,6$ & $149,3 \pm 43,6$ & $-0,82$ & 0,43 \\
\hline
\end{tabular}

Média e desvio-padrão, estatística t e valor p referentes às variáveis segundo grupo. Emissão da vogal sustentada /a/.

Tabela 4 - Porcentagem de alterações dos componentes da fala nos grupos com Doença de Parkinson (DP) e Grupo Controle

\begin{tabular}{|c|c|c|c|}
\hline & \multicolumn{3}{|c|}{ Grupo } \\
\hline & \multicolumn{2}{|c|}{ Parkinson $(n=21)$} & \multirow{2}{*}{$\begin{array}{c}\text { Normal }(n=10) \\
N^{\circ}\end{array}$} \\
\hline & № & $\%$ & \\
\hline Fonação & 18 & 85,7 & - \\
\hline Respiração & 4 & 19,0 & - \\
\hline Articulação & 8 & 38,1 & - \\
\hline Ressonância & 5 & 23,8 & - \\
\hline Prosódia & 7 & 33,3 & - \\
\hline
\end{tabular}

\section{DISCUSSÃO}

A sistematização na avaliação de sinais e sintomas é fator essencial no estabelecimento do diagnóstico e planejamento terapêutico. A utilização de protocolos é imperativa tanto no que se refere à eficiência como na reprodutibidade dos achados e subsequente análise. A versão, adaptação e normatização devem ter sua aplicação aferida pela utilização tanto em indivíduos normais como naqueles portadores de distúrbios.

A tradução e adaptação do protocolo cedido pelo Professor Wolfrang Ziegler, apresentada neste estudo, reforça a necessidade de instrumentos capazes de fornecer dados qualitativos e quantitativos, que auxiliem a prática clínica.

A aplicação do protocolo já traduzido e adaptado às particularidades fonéticas e fonológicas do português falado no Brasil demonstrou que o distúrbio vocal foi a anormalidade mais frequente, encontrada em $89 \%$ dos pacientes com Doença de Parkinson. Dado este que corrobora os achados da literatura que encontraram o distúrbio vocal como sendo o mais frequente nessa população ${ }^{6}$.

A disfunção laríngea tem sido apontada como característica comum da disartria hipocinética, sendo o distúrbio da fonação frequentemente observado nos estágios iniciais da doença. As características de disfunções laríngeas mais prevalentes em nosso estudo foram o tempo máximo de fonação $(66,7 \%)$ e a reduzida intensidade vocal $(61,9 \%)$.

O tempo máximo de fonação (TMF) mostrou-se significantemente mais curto em indivíduos com DP quando comparados ao grupo controle, dados estes que corroboram com o estudo de Midi et $\mathrm{al}^{16}$.

O elevado percentual de alterações vocais $(85,7 \%)$ e articulatórias $(38,1 \%)$ registrados no atual estudo confrontam com o percentual relativamente baixo $(23,8 \%)$ de alterações de ressonância. Esses resultados repetem aqueles apresentados por Logemann ${ }^{6}$ com distúrbios articulatórios em $45 \%$ dos pacientes com DP e somente $10 \%$ com disfunção de ressonância. Ackermann ${ }^{12}$ chama a atenção para o fato de que na DP os distúrbios articulatórios relacionados ao tempo de fala não 
são alterações evidentes. Os pacientes analisados apresentaram redução no movimento e amplitude dos articuladores. Neste estudo, da mesma forma, este distúrbio mostrou-se pouco frequente $(38,1 \%)$.

Quanto à análise acústica os parâmetros com diferença estatisticamente significantes foram jitter, shimmer, SFF e PA. Na literatura observou-se que as medidas jitter e shimmer encontram-se freqüentemente alteradas na DP ${ }^{10}$.

Levando em conta todos esses achados é possível estabelecer a hipótese de que a menor incidência de distúrbios articulatórios em nossos pacientes estava relacionada ao estágio da doença. No entanto, são necessários outros estudos com maior número de sujeitos correlacionando o grau de comprometimento, tempo de evolução, faixa etária e gênero para que essa relação seja mais bem estabelecida. Estudos destacam que embora o fator inteligibilidade de fala seja uma característica frequentemente alterada em pacientes com DP esta ainda não foi bem descrita quanto à sua relação com a gravidade da doença, duração ou fenótipo motor ${ }^{17}$.

No estudo que serviu de base para este protocolo foram analisados 12 pacientes com Doença de Parkinson, incluindo outros diagnósticos como depressão, demência e diferentes graus de alterações de compreensão, sem qualquer preocupação com estagiamento da doença, o que inviabilizou a comparação com resultados da pesquisa ${ }^{14}$. No entanto, a utilização do protocolo original em outro estudo com sujeitos disártricos pós-acidente vascular encefálico confirma a importância da existência de parâmetros quantitativos e qualitativos que possibilitem a análise confiável dos achados clínicos ${ }^{18}$.

O acompanhamento clínico de pacientes com quadros disártricos, especialmente relacionados ao processo de identificação e avaliação, tem ocorrido, principalmente, por meio da análise perceptiva auditiva e acústica da fala. Esses parâmetros, segundo a literatura, são complementares entre $\mathrm{si}^{10}$. O estabelecimento de protocolos específicos que contemplem as variações e peculiaridades da língua portuguesa falada no Brasil é visto como ferramenta importante na avaliação da disartria por diferentes profissionais que atuam com essa população.

Os dados obtidos com a aplicação do protocolo proposto neste estudo fornecem parâmetros de auxílio para a prática clínica relacionada tanto à avaliação, como à terapia e prognóstico na disartria.

\section{CONCLUSÃO}

O presente estudo fornece protocolo de fácil aplicação para avaliação da disartria. O protocolo é uma ferramenta importante na atuação clínica. No entanto, faz-se necessária a continuidade dos estudos visando possíveis modificações no protocolo e aplicação deste em maior espectro de sujeitos e distúrbios neurológicos para validação plena do mesmo.

\begin{abstract}
Purpose: to translate and adapt the protocol developed by German researchers, adjusting it to the phonetic and linguistic characteristics of Brazilian Portuguese. Observe the amended speech components in a group of patients with Parkinson's disease, and comparing them with normal subjects in the same age. Method: twenty one patients with neurological diagnosis of Parkinson's disease in the Hoehn \& Yarh stages (2-3), and 10 control subjects were assessed. The protocol was translated, adjusted and applied to the subjects of both control and Parkinson's disease group, assessing the breathing, phonation, resonance, articulation, prosody and acoustic analysis. Results: in the dysarthria evaluation, the protocol showed to be simple and it presented a fast clinical application. In the subjects with Parkinson's disease, we observed alterations in both phonation (85.9\%) and articulation (42.9\%). Conclusion: the study demonstrated that the protocol is an efficient tool for dysarthria assessment in patients with Parkinson's disease.
\end{abstract}

KEYWORDS: Dysarthria; Parkinson Disease; Evaluation 


\section{REFERÊNCIAS}

1. Murdoch DE. Disartria: uma abordagem fisiológica para avaliação e tratamento. São Paulo: Lovise; 2005.

2. Oliveira CR, Ortiz KZ, Vieira MM. Disartria: estudo da velocidade de fala. Pró-Fono. 2004; 16(1):39-48. 3. Palermo S, Basto ICC, Mendes MFX, Tavares EF, Santo DCL, Ribeiro AFC. Avaliação e intervenção fonoaudiológica na doença de Parkinson. Análise clínica-epidemiológica de 32 pacientes. Rev. bras. neurol. 2009; 45(4): 17-24.

4. Hoehn MM, Yahr MD. Parkinsonism: onset, progression and mortality. Neurology. 1967; 17:427-42.

5. Skodda S, Rinsche H, Schlegel U. Progression of dysprosody in Parkinson's disease over time a longitudinal study. Mov Disord. 2009; 24(5): 716-22.

6. Logemann JA, Fisher HB, Bosches B, Blonsky E. Frequency an cooccurrence of vocal tract dysfunctions in the speech of a large sample of Parkinson's patients. J Speech Hear Disord. 1978; 43:47-57.

7. Walshe $M$, Miller $N$, Leahy $M$, Murray $A$. Intelligibility of dysarthric speech: perceptions of speakers and listeners. Int $\mathrm{J}$ Lang Commun Disord. 2008; 43(6): 633-48.

8. Erqun A, Oder W. Oral diadochokinesis and velocity of narrative speech: a prognostic parameter for the outcome of diffuse axonal injury in severe head trauma. Brain Inj. 2008; 22(10):773-9.

9. Auzou P, Kouadio V, Rigaux P, Ozsancak C. A perceptual analysis of dysarthria following cranial trauma. Rev Neurol. 2007;163(12):1200-8.

10. Ortiz KZ, Carrillo L. Comparação entre as análises auditiva e acústica nas disartrias. Rev Soc Bras Fonoaudiol. 2008; 13(4):325-31.
11. Walshe $M$, Miller $M$, Leahy M, Murray $A$. Intelligibility of dysarthric speech: perceptions of speakers and listeners. Int J Lang Commun Disord. 2008; 6:633-48.

12. Ackermann $\mathrm{H}$, Ziegler W. Articulatory déficits in parkinsonian dysarthria: an acoustic analysis. $J$ Neurol. 1991; 54:1093-8.

13. Ortiz KZ. Avaliação das disartrias. In: Ortiz KZ. Distúrbios neurológicos adquiridos: fala e deglutição. São Paulo: Manole; 2006. p.73-83.

14. Ziegler W, Hartmann E, Hoole P, Cramon DV. Entwicklung von diagnostischen Standards und von Therapieleitlinien für zentrale Stimm und Sprechstörungen (Dysarthrophonien)- Forschung des Projektträgers 2/90. Gessellschaft für Strahlenund Umweltforshung mbH München; 1990.

15. Montagnoli AN. Análise residual do sinal da voz [dissertação]. São Carlos (SP): Universidade de São Paulo; 1998.

16. Midi I, DoganM, Koseoglu M, Can G, Sehitoglu MA, Gunal DI. Voice abnormalities and their relation with motor dysfunction in Parkinsons disease. Acta Neurol Scand 2008; 117: 26-34.

17. Miller N, Allcock L, Jones D, Noble E, Hildreth AJ, Burn DJ. Prevalence and pattern of perceived intelligibility changes in Parkinson's disease. J Neurol Neurosurq Psychiatry. 2007; 78(11): 1188-90.

18. Urban PP, Wicht S, Keilmann A, Stoeter P, Hapf $\mathrm{HC}$, Dieterich M. Left-hemispheric dominance for articulation: a prospective study on acute ischaemic dysarthria at different localizations. Brain. 2006; 129:767-77.

http://dx.doi.org/10.1590/S1516-18462011005000030

RECEBIDO EM: 10/08/2010

ACEITO EM: 06/10/2010

Endereço para correspondência:

Priscila Watson Ribeiro

Campus Universitário da Unesp

Botucatu - SP

CEP: 18618-000

E-mail: priwtr@yahoo.com.br 


\section{Anexo 1}

\section{PROTOCOLO DE AVALIAÇÃO DA DISARTRIA}

Nome:

Idade: Profissão: $R G$ :

Fumante: ( ) Não ( ) Sim Tempo HDNeurológica:

Data da Internação:: Escolaridade: Imagem: Data Avaliação:

\section{I - RESPIRAÇÃO}

- Velocidade: Ciclos/minuto ( $\mathrm{nl}-12$ a $20 \mathrm{c} / \mathrm{min}$ ).

- Tempo máximo (nl - 10 a 20 segundos) de fonação - observação da manutenção tonal:
la/ s
/i/ S
/s/ $s$
$|z|$ S

OBS:

- Relação s/z : $(n l-0,7$ a 1,3)

- Palavras por expiração - contagem espontânea de números (40 a 1):

Respiração: Análise indica comprometimento de grau:

0---1---2---3---4---5---6

(grau 0 ausência; 6 grave)

\section{II - FONAÇÃO}

- Qualidade Vocal:

Normal

Pastosa

Trêmula

Rouca

Áspera

Soprosa

\section{- Altura Vocal:}

Adequada

Grave

Aguda

\section{- Ataque Vocal:} Isocrônico Brusco Aspirado

( )

( )
- Intensidade Vocal (int):

Adequada ( )

Alta

Baixa

( )

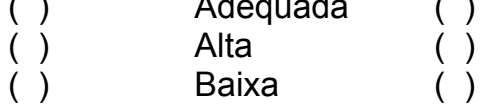

- Variação da qualidade vocal:

Estável ( )
Instável

Voz: Análise indica comprometimento de grau:

0---1---2----3---4---5---6

(grau 0 ausência; 6 grave) 


\section{III - RESSONÂNCIA}

- Movimento velar - /a/ e /â/ alternadamente:

- Movimentação parede faríngea - ka ka:

Adequada

( )

Mínima

( )

Adequada

Mínima

( )

Ausente

Ausente ( )

( )

- Emissão nasal (espelho):

Mamão x papai / pau x mau

Papai pediu pipoca.

A fita de filó é verde.

Vovó viu a uva.

Amanhã mamãe amassará mamão.

Normal ( ) Hipernasalidade: leve ( ) grave ( ) Hiponasalidade: leve ( ) grave ( )

Ressonância: Análise indica comprometimento de grau:

0---1---2---3----4---5---6

(grau 0 ausência; 6 grave)

\section{IV - ARTICULAÇÃO}

- Movimentos Lábio (i/u e pa) - espontâneos e forçados:

Normal

( )

Alterado

( )

- Língua (ka/ta - velocidade crescente):

Normal

( )

Alterado

( )

- Mandíbula - abertura:

Normal ( )

Alterado ( )

- Desenho para paciente descrever/ conversa espontânea:

Respiração

Articulação

Ressonância

- Leitura de monossílabos e de frases ( $n^{\circ}$ : ):

\section{Plosivas:}

Banco

Dedo

Porco

Batata

Normal ( ) Alterado( )
Plosivos e nasais, na emissão de palavras e fora:

\section{P TK $\quad$ Cama $\quad$ Balão}

B DG Pão Caminhão

$\mathrm{m} / \mathrm{n} / \mathrm{nh} \quad$ Mão

Normal ( ) Alterado( )

Vogais isoladas e vogais nas palavras:

\section{A EIOU}

Meia Pia

Bóia Baú

Normal ( ) Alterado( ) 
Líquidas:

$\begin{array}{ll}\text { Lápis } & \text { Milho } \\ \text { Lua } & \text { Olho } \\ \text { Bolo } & \text { llha } \\ \text { Normal ( ) } & \text { Alterado（） }\end{array}$

\section{Encontros Consonantais}

Prato Blusa

Flores Fralda

Normal ( ) Alterado( )

Diadococinesia (repetição espontânea velocidade crescente*):

Pataka*

Badaga

Fasacha

Normal ( ) Alterado( )

- Precisão articulatória

0---1---2---3---4---5---6

(grau 0 - ininteligível; 6 - inteligível)

Articulação: Análise indica comprometimento de grau:

0---1---2---3---4----5---6

(grau 0 ausência; 6 grave)

\section{V - PROSÓDIA}

- Entonação (repetição sem indução de entonação é permitida):

1- Afirmação:

É proibido fumar aqui.

Normal ( ) Alterado ( )

2- Interrogação:

Você gostaria de comprar bolo ou sorvete?

Normal ( ) Alterado ( )

3- Exclamação:

Maria chegou!

Normal ( ) Alterado ( )

- Velocidade:

Normal ( ) Alterado ( )

- Pausas na fala

0---1---2----3---4---5---6

(grau 0 - sem pausas; 6 muitas pausas)

Prosódia: Análise indica comprometimento de grau:

0---1---2---3---4---5---6

(grau 0 ausência; 6 grave)

\section{DISARTRIA}

$\begin{array}{ll}\text { ( ) LEVE } & \text { (1 a } 10 \text { pontos) } \\ \text { ( ) MODERADA } & \text { (11 a } 20 \text { pontos) } \\ \text { ( ) GRAVE } & \text { (21 a } 30 \text { pontos) }\end{array}$

